\title{
Cannabis: à quel saint se vouer?
}

\section{Daniele Fabio Zullino ${ }^{a}$,} Riaz Khan ${ }^{b}$, Yasser Khazaal ${ }^{b}$

a PD Dr, médecin chef de service

b Dr, médecin adjoint

Service d'addictologie, Département de psychiatrie, Hôpitaux Universitaires de Genève

1 Zullino DF, Kurt H, Broers B, Drexler A, Graf HP, Khazaal Y, et al. Swiss psychiatrists beliefs and attitudes about cannabis risks in psychiatric patients: ideologically determined or evidence-based? Community Ment Health J. 2008; 44(2):86-96.

2 Zobel F, Gervasoni JP, Jeannin A. Enquête auprès des partenaires de l'Office fédéral de la santé publique dans le domaine de la toxicomanie (drogues illégales) Lausanne: IUMSP; 1999.

3 Hämmig R, Savary JF, Theunert M. Replik. Bull Méd Suisses. 2008; 89(26):1181-2.

4 Hämmig R, Savary JF, Theunert M. Politique cannabis: ni diabolisation, ni banalisation. Bull Méd Suisses. 2008;89(17):748-52.

5 Beutler-Hohenberger D, Dunant JH, Thévot F, Jundt U. Cannabispolitik: und sie dreht sich doch! Bull Méd Suisses. 2008;89(26-27):1179-81.

6 Kant I. Grundlegung zur Metaphysik der Sitten. Göttingen: Vandenhoek \& Ruprecht; 2004.

Correspondance:

PD Dr Daniele Zullino

Hôpitaux Universitaires de Genève

Département de Psychiatrie

Service d'addictologie

Rue verte 2

CH-1205 Genève

Tél. 0223725560

Fax 0223281760

daniele.zullino@hcuge.ch
Nous nous sommes récemment intéressés aux attitudes des psychiatres suisses vis-à-vis des consommations de cannabis de leurs patients [1]. Nous avons eu l'occasion de questionner 82 collègues concernant leurs appréciations des effets du cannabis sur le développement de différents troubles psychiatriques, ainsi que sur leurs attitudes face à des patients consommateurs présentant ces différents troubles. Trois clusters sortaient des analyses:

1. dans le premier groupe ("prohibitionistes», 22 collègues) se trouvaient des médecins qui partageaient la conviction que le cannabis causait tous les troubles considérés. Aussi, ils prohibaient systématiquement l'usage de cannabis quelque soit le trouble psychiatrique en question;

2. les médecins du deuxième groupe («causalistes», 46 médecins) envisageaient que le cannabis pouvait être un facteur causal de la schizophrénie seulement. En conséquence logique ils tentent de dissuader plus particulièrement ces patients de fumer du cannabis;

3. le troisième groupe («les libéralistes prudents», 14 médecins) était composé de collègues qui doutaient fortement d'un lien causal entre cannabis et troubles psychiques. Ces derniers optaient pour une position plus indulgente.

Les positions (et même les croyances) d'une proportion importante des psychiatres suisses questionnés reflètent de façon importante la polarisation des attitudes (prohibition catégorique vs. attitude permissive) retrouvée dans la population générale [2]. Du moment que les avis sont si partagés, il est évident que pour au moins une large partie des médecins la prise de position ne peut pas être basée sur des évidences scientifiques. Elle est donc probablement basée sur des visions idéologiques.

Comme s'il fallait en alimenter l'évidence, les argumentaires proposés récemment par Hämmig et al. d'une part [3, 4] ainsi que par BeutlerHohenberger et al. [5] d'autre part permettent de corroborer de façon plutôt caricaturale la thèse de la difficulté des médecins, tout du moins dans certaines polémiques, à ne pas distancier convictions idéologiques de méthodes basées sur les évidences. Ce phénomène ne devrait donc pas trop étonner si on considère le sujet des addictions particulièrement empreint de références

\section{Résumé}

Les positions d'une partie importante des psychiatres suisses vis-à-vis du cannabis tendent à refléter de façon assez fidèle la polarisation des attitudes (prohibition catégorique vs. attitude permissive) retrouvée dans la population générale. Cela laisse à penser que pour une partie des médecins, la position soutenue n'est pas basée sur des évidences scientifiques. Elle est donc probablement basée sur des visions idéologiques.

Certains articles publiés dans cette rubrique (Hämmig et al. et Beutler-Hohenberger et al.) reflètent clairement ce phénomène, en se basant sur le mélange d'une logique déontologique et des propos évoquant la recherche d'évidences scientifiques. En soi, les deux approches, déontologique et basée sur les preuves, sont possibles et ont leur place dans ce débat, mais leur mélange peut être problématique.

culturelles morales. Pas surprenant vu qu'on débat non pas de comprendre mais d'interdire ou de permettre. Le plus souvent, deux catégories d'approches sont distinguées dans un tel débat: l'approche déontologique versus l'approche utilitariste.

\section{L'approche déontologique}

L'approche déontologique (du terme grec deon: l'indispensable, «le doit-être», le devoir) part du présupposé que «le bien» est quelque chose de prédéfini indépendamment de la prise de conscience humaine et du discernement éclairé. En d'autres termes, ce qui est bien est établi indépendamment de nouveaux éclairages ou des modifications des conséquences. A cet égard, il incombe à l'homme doué de raison d'agir toujours en vertu de ce qui est dû, même si cette action a des conséquences négatives. Le représentant les plus illustre de ce courant est Emmanuel Kant avec son impératif catégorique [6]. Selon cette conception, les actes de l'homme ne devraient pas seulement servir de moyen pour atteindre un 
but, mais être intrinsèquement bien. L'impératif catégorique est illustré par exemple par la formulation suivante de Kant: «Agis selon des maximes qui puissent en même temps se prendre ellesmêmes pour objet comme lois universelles de la nature.» On trouve une illustration populaire de cette conception dans le dicton: «Imagine-toi que c'est permis à tout le monde!». Dans notre cas concret le dicton se traduirait par: «Imaginetoi si tout le monde avait le droit de fumer du cannabis.» Kant considérait que l'homme peut établir par sa seule capacité à raisonner le bien, sans avoir besoin de faire appel aux circonstances ou aux expériences.

\section{L'utilitarisme}

L'utilitarisme est une approche qui considère que ce qui est «utile» est bon. Un de ces concepteurs majeurs est John Stuart Mill, selon lequel «le bien» se détermine par l'utilité sociale comme l'illustre sa formule: «le plus grand bonheur du plus grand nombre». Une des caractéristiques des approches utilitaristes est d'éviter des à priori sur ce que devraient être les règles de vie en société. Dans cette perspective «le bien» se définit en fonction des conséquences attendues et/ou observées, tenant notamment compte des variations de contexte. Pour se déterminer, l'homme doit donc apprécier ces paramètres. C'est dans cet aspect des choses que les procédures basées sur les évidences s'inscrivent.

La médecine basée sur l'évidence (evidencebased medicine $=$ EBM) est définie comme «l'utilisation rigoureuse et judicieuse des meilleures données disponibles lors de prise de décisions concernant les soins à prodiguer à des patients individuels» [7]. C'est une démarche qui nécessite les étapes suivantes: 1 . formuler une question explicite à partir d'un problème donné; 2 . rechercher les références les plus pertinentes; 3. évaluer les niveaux de validité et utilité des références; 4. intégrer ces données dans le contexte d'élaboration de la réponse à la question posée; 5 . évaluer l'impact de l'application de la réponse donnée.

Ce qui frappe à la lecture des articles de Hämmig et al. [3, 4] et Beutler-Hohenberger et al. [5] est le panachage cocasse des deux approches. Bien qu'il soit instructif d'analyser sous ces angles chacun des passages des trois articles, nous proposons de nous limiter à quelques exemples.

\section{Exemples de positionnement déontologique}

A la page 1182 Hämmig et al. [4] considèrent la loi sur les stupéfiants comme une manière d'infliger des dommages aux personnes, ce qui serait contraire à un point de vue d'éthique médicale $\mathrm{du}$ «noli nocere». Par la suite une distinction est faite entre «vice publique» et «vice privé», la pénalisation du premier étant considérée comme éthiquement justifiable, alors que celle du vice privé ne le serait pas.

Ce passage illustre joliment une démarche déontologique qui n'a, en principe, aucunement besoin d'étayage par des mesures basées sur les évidences. Le «bien» est trouvé par pur raisonnement et devrait par principe devoir s'appliquer à toutes les situations imaginables.

De l'autres côté du débat, à la page 1179 , Beutler-Hohenberger et al. [5] postulent que dès que l'on se solidarise avec les activistes du chanvre, on ne serait plus libre d'idéologies! Selon eux, l'initiative reflèterait un courant de pensée «Sex, Drugs and Rock'n'roll» qui propagerait une idéologie destructrice.

Là aussi, et sans jugement sur le contenu des propos, on peut constater la même démarche déontologique basée sur le seul raisonnement.

Finalement, les deux groupes pourraient poursuivre leur débat en élaborant encore ces positions déontologiques, en développant leur raisonnement et les valeurs qui les sous-tendent. Nul besoin de s'efforcer à chercher, lire, appréhender et transmettre des éléments d'évidences scientifiques, les évidences ne pouvant dans une démarche déontologique changer les conclusions. Les deux groupes se sont tout de même efforcés à étayer leurs propos par des éléments issus de l'EBM.

\section{Exemples de positionnements EBM}

A la page 750 Hämmig et al. [3] constatent: «Etant donné que le tronc, centre de régulation des fonctions vitales comme la respiration, est peu doté de ce type de récepteurs, les intoxications mortelles à base de cannabis sont pour ainsi dire exclues. Jusqu'à ce jour, aucun cas de décès par effet direct du cannabis n'est décrit.»

L'appréciation de cet énoncé pourrait se faire à la lecture des étapes de l'EBM (voir ci-dessus). Concernant l'étape 1, la question n'a pas été explicitement formulée, mais peut clairement être comprise de part la suite de l'énoncé. Pour ce qui est de l'étape 2, la recherche des meilleures évidences semble quelque peu négligée. En effet, s'il y a effectivement peu de récepteurs CB1 dans le tronc, il n'en est pas moins qu'il y en a [8], et que de plus, des cannabinoïdes peuvent induire une inhibition des fonctions respiratoires $[8,9]$ par leur effet sur ces mêmes récepteurs [8]. Par ailleurs, de multiples publications relatent des cas d'arythmies, d'infarctus et d'accidents vasculaires cérébraux attribués au cannabis, dont plusieurs cas mortels (p.ex. [10-12]). 
En considérant que la question posée implicitement dans l'étape 1 était «le cannabis peut-il induire des intoxications fatales?», la réponse apportée dans l'étape 4 ne tient pas compte de plusieurs paramètres potentiellement impliqués dans de tels incidents. D'emblée tout le raisonnement est basé sur un effet potentiel sur le bulbe. Des effets périphériques, étant par exemple ignorés. Par ailleurs, l'effet des cannabinoïdes n'est de loin pas limité aux seuls récepteurs cannabinoïdes [13]. Par ailleurs, même l'absence de récepteurs n'empêcherait pas forcément un effet sur les fonctions vitales du tronc. L'alcool en est l'exemple par excellence. Jusqu'à présent on ne connaît pas de récepteurs à l'éthanol, mais ses effets dépressogènes respiratoires à travers des structures du tronc peuvent être considérés comme bien documentés (p.ex. [14-16]).

Ce n'est donc pas l'évidence qu'il n'y ait pas de récepteurs $\mathrm{CB}$ dans le tronc cérébral qui est contestable, mais les déductions faites. Notamment on ne peut pas dire - sur la base des meilleures évidences actuellement à disposition que «jusqu'à ce jour, aucun cas de décès par effet direct du cannabis n'est décrit».

Le même type d'observations peut être fait pour l'article de Beutler-Hohenberger et al. [5]. Ainsi à la page 749 les auteurs affirment que concernant le développement cognitif l'article de Hämmig et al. omettrait certaines données essentielles. Ils citent deux références qui auraient conclu que même une consommation modérée de cannabis durant la grossesse conduit à des altérations irréversibles du cerveau fotal, qui conduiraient à des perturbations du développement émotionnel des enfants.

En considérant la démarche EBM précédemment décrite, les auteurs ont également de manière implicite formulés leur question qu'on suppose être: le cannabis altère-t-il le développement fotal du cerveau de façon à perturber l'évolution psychique de l'enfant? Les articles mis en référence rapportent des résultats incontestablement intéressants sur le rôle des récepteurs CB1 dans la guidance axonale (axon guidance) [17] et la plasticité de l'emotional learning [18]. Concernant l'étape 2, celle de la recherche de la meilleure évidence, on peut déjà se poser la question du choix de ces articles. C'est cependant l'étape 4 qui interpelle le plus. En effet, bien que le dernier alinéa du travail de Berghuis et al. [17] spécule sur les éventuels effets d'une exposition embryonnaire aux THC sur le développement de la cognition et du comportement, cette hypothèse de Berghuis et al. est d'emblée rapportée comme une conclusion par Beutler-Hohenberger et al. alors que les résultats de l'étude corroborent simplement l'implication des récepteurs CB1 dans la guidance axonale. Quant aux résultats de l'étude animale publiée par Laviolette et Grace [18], ils corroborent simplement une participation des récepteurs CB1 dans certains aspects de l'emotional learning (apprentissage des associations conditionnées dans un contexte émotionnel). Malgré l'intérêt de ces résultats, ils ne permettent pas de conclure à un impact du cannabis consommé durant la grossesse sur le développement émotionnel des enfants. Au vu de ce qui précède, il semble que le contenu des articles choisis ne permette pas vraiment de répondre à la question posée.

\section{Discussion}

L'étude sur les attitudes des psychiatres suisses vis-à-vis du cannabis avait révélé des positionnements différents et tranchés, qui en conséquence ne pouvaient pas être toutes basées sur des évidences scientifiques. Le débat récemment publié dans cette rubrique est le reflet de ce constat. En effet les deux parties qui se sont jusqu'à présent exprimés se reprochent mutuellement de prendre des positions idéologiques malgré les déclarations faites de se baser sur les évidences scientifiques. Pourrait-on dire que les deux groupes ont raison?

On retrouve d'ailleurs chez les deux parties des passages s'appuyant sur une lecture déontologique. En soi, cette logique a sa place dans les débats de société et les articles auraient probablement gagné à être développés sous cet angle. En choisissant clairement cette option, il n'y aurait pas besoin de charger la discussion avec des évidences.

Toutefois, les deux groupes ont tenté de soutenir leurs thèses avec des références scientifiques. Comme l'illustrent ces quelques exemples choisis, la rigueur des deux groupes est perfectible. De manière générale, si on devait choisir l'option EBM dans cette discussion, il apparaît qu'une meilleure diligence dans l'application des règles de l'EBM est de mise pour éviter des débats peu convaincants.

Ceci est d'autant plus ennuyeux que les deux groupes soutiennent leurs thèse au nom des «spécialistes de l'addiction» [4] ou des «addictologues orientés abstinences» (abstinenzorientierte ... Drogenfachleute) [5]. Il se peut que les auteurs représentent la position des associations dont ils se réclament (en supposant qu'ils en aient le mandat), mais en aucun cas ils peuvent prétendre s'exprimer au nom de l'ensemble des spécialistes. Les trois articles de Hämmig et al. et Beutler-Hohenberger et al. ont au moins eu le mérite de lancer un débat qui sera sans doute fructueux et passionnant, d'autant plus qu'on s'en donnera les moyens. 